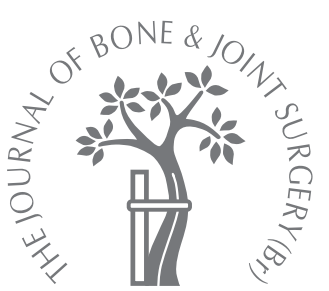

A. Pradhan,

Y. C. Cheung,

R. J. Grimer,

D. Peake,

O. A. Al-Muderis,

J. M. Thomas,

M. Smith

From the Royal

Orthopaedic Hospital,

Birmingham, England

A. Pradhan, MBChB(Hons), MBedSc, Specialist Registrar Staffordshire General Hospital Weston Road, Stafford ST16 3SA, UK.

I. C. Cheung, MBChB,

Foundation Year 2

Queen's Hospital, Belvedere

Road, Burton-on-Trent DE13

ORB, UK

- R. J. Grimer, FRCS(Orth), Consultant Orthopaedic

Oncologist

D. Peake, FRCR, Consultant

Oncologist

Oncology Department

Royal Orthopaedic Hospital,

Birmingham B31 2AP, UK.

- O. A. Al-Muderis, BSc(Hons)

Sarcoma Unit Data Manager

I J. M. Thomas, FRCS,

Consultant General Surgeon

Sarcoma Unit

Royal Marsden Hospital,

Fulham Road, London SW3

6JJ, UK.

M. Smith, FRCS(Orth),

Consultant Orthopaedic

Surgeon

Guy's and St Thomas NHS

Foundation Trust, Guy's

Hospital, St Thomas Street

London SE1 9RT, UK.

Correspondence should be sent to MrR. J. Grimer; e-mail: rob.grimer@roh.nhs.uk

(C2008 British Editorial Society of Bone and Joint Surgery doi:10.1302/0301-620X.90B2. $19601 \$ 2.00$

$J$ Bone Joint Surg $[\mathrm{Br}]$

2008;90-B:209-14.
Received 25 April 2007;

Accepted after revision 18

October 2007

\title{
Soft-tissue sarcomas of the hand
}

\author{
ONCOLOGICAL OUTCOME AND PROGNOSTIC FACTORS
}

We have investigated the oncological outcome of 63 patients with soft-tissue sarcomas of the hand managed at three major centres in the United Kingdom. There were 44 males and 19 females with a mean age of 45 years (11 to 92). The three most common diagnoses were synovial sarcoma, clear cell sarcoma and epithelioid sarcoma. Local excision was carried out in 45 patients $(71 \%)$ and amputation in $18(29 \%)$. All those treated by amputation had a wide margin of excision but this was only achieved in $58 \%$ of those treated by local excision. The risk of local recurrence was $6 \%$ in those treated by amputation compared with $\mathbf{4 2} \%$ for those who underwent attempted limb salvage. An inadequate margin of excision resulted in a 12 times greater risk of local recurrence when compared with those in whom a wide margin of excision had been achieved. We were unable to demonstrate any role for radiotherapy in decreasing the risk of local recurrence when there was an inadequate margin of excision. Patients with an inadequate margin of excision had a much higher risk of both local recurrence and metastasis than those with wide margins. The overall survival rate at five years was $87 \%$ and was related to the grade and size of the tumour and to the surgical margin.

We have shown that a clear margin of excision is essential to achieve local control of a soft-tissue sarcoma in the hand and that failure to achieve this results in a high risk of both local recurrence and metastastic disease.

The management of soft-tissue sarcomas of the hand is a matter of controversy and presents challenges to both the surgeon and oncologist. ${ }^{1,2}$ Most soft-tissue sarcomas of the limbs are currently treated by wide excision and adjunctive radiotherapy. ${ }^{3-6}$ However, this approach is difficult to apply to the hand because of its anatomy and the lack of readily expendable soft tissue (Fig. 1). The sacrifice of important nerves, blood vessels and tendons to gain an adequate margin may result in significant functional loss. ${ }^{7,8}$ Alternatively, aiming to preserve function by just removing the tumour itself may compromise the outcome. ${ }^{9}$ Furthermore, it has been reported that radiotherapy to the hand is poorly tolerated and associated with an increased risk of long term toxicity and poor outcome. , $^{2,10}$

Few studies ${ }^{11-14}$ have been carried out which look specifically at the surgical treatment of softtissue sarcomas of the hand and its outcome. We wished to assess whether limb-sparing surgery with close margins would result in a high rate of local recurrence and whether this would compromise survival.

We report a large series of patients with softtissue sarcomas of the hand treated at three centres in the United Kingdom (The Royal Orthopaedic Hospital, Birmingham, The Royal Marsden Hospital, London, and St Thomas's Hospital, London). The treatment philosophy was the same at each centre. The tumour was excised with a clear margin whenever possible. Radiotherapy was used if a clear margin could not be achieved without a major loss of function. Primary amputation was carried out only when tumours were large and complete clearance could not be obtained with more conservative surgery. Chemotherapy was only used if the tumour was chemosensitive. We assessed the oncological outcome of these patients and analysed the prognostic factors for local control and patient survival. We did not assess their functional outcome.

\section{Patients and Methods}

Between 1990 and 2003, the three centres treated 87 patients with soft-tissue sarcomas of the hand distal to the wrist. These were identified from the centres' sarcoma database and represented approximately $2 \%$ of the limb and trunk soft-tissue sarcomas seen at these centres. We excluded ten patients who were 


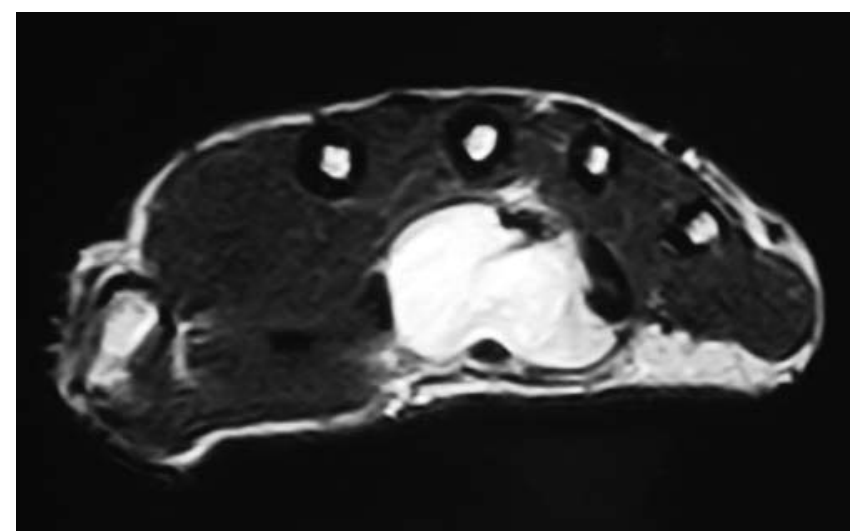

Fig. 1

MR scan of the dominant hand of a 50 -year-old man who presented with features typical for carpal tunnel syndrome. The MRI was carried out because of slight swelling in the palm and showed a $4 \mathrm{~cm} \times 2 \mathrm{~cm}$ mass because of slight swelling in the palm and showed a $4 \mathrm{~cm} \times 2 \mathrm{~cm}$ mass
thought to be a lipoma. It was 'shelled out' at the time of carpal tunnel release and was found to be a biphasic synovial sarcoma. In order to obtain clear margins the patient elected to have a below elbow amputation. There was extensive residual tumour found in the amputation specimen.

only seen for consultation, four patients with a recurrence after treatment elsewhere and ten with metastases at diagnosis. We included patients who had undergone an inadvertent biopsy or excision biopsy. This left 63 patients in the study group.

The following data were collected retrospectively from the case records or the tumour databases: age at surgery, gender, maximum tumour dimension $(\mathrm{cm})$, histological type and grade (using the Trojani system ${ }^{15}$ ), surgical margin, use and method of adjuvant radio- or chemotherapy, time to local recurrence after surgery, time to systemic recurrence and overall survival. Tumour size was taken as the greatest dimension of the tumour as estimated from pathology reports following resection, or in some cases from MRI evaluation. The margins of excision were classified either as 'clear', with no tumour at the edge, or 'involved' where tumour extended to the edge. A radical amputation was defined as one occurring above the wrist joint while a partial amputation included digital and ray amputations. All patients underwent staging with a CT scan of the chest at the time of initial diagnosis and were subsequently followed up with regular chest radiographs to detect metastatic disease. Statistical analysis. Differences between groups were assessed using the chi-squared test or Student's $t$-test. Overall survival and the rate of local recurrence was calculated using the Kaplan-Meier method and the impact of prognostic factors was assessed using the log rank test. ${ }^{16}$ Multivariate analysis was performed using Cox's proportional hazard method, ${ }^{17}$ variables were chosen using a forward conditional stepwise approach. Relative risks were calculated using a proportional hazards model with only the noted covariate in the model.
Table I. Distribution of histological diagnoses

\begin{tabular}{ll}
\hline Diagnosis & Number of cases $(\%)(\mathbf{n}=\mathbf{6 3})$ \\
\hline Clear cell sarcoma & $11(17.4)$ \\
Epithelioid sarcoma & $11(17.4)$ \\
Synovial sarcoma & $10(16)$ \\
Fibrosarcoma & $7(11.1)$ \\
Leiomyosarcoma & $3(5)$ \\
Liposarcoma & $3(5)$ \\
Dermatofibrosarcoma & $2(3.1)$ \\
Others & $16(25)$ \\
\hline
\end{tabular}

Significance was set at $\mathrm{p}<0.05$ for two-sided tests. Survival time was calculated from the time of diagnosis. Endpoints were taken as the time of death or the last documented time the patient was known to be alive. Patients who died of unrelated causes were considered to have been censored.

\section{Results}

Patient demographics and tumour characteristics. There were 44 males and 19 females in the series with a mean age of 45 years (11 to 92 ). The mean follow-up was 50 months (3 to 180).

Clear cell sarcoma $(11$ of $63,17.4 \%)$ and epithelioid sarcoma ( 11 of $63,17.4 \%$ ) were the most common diagnoses, followed by synovial sarcoma ( 10 of $63,16 \%$ ), with other diagnoses being less common (Table I). The mean tumour size was $4 \mathrm{~cm}(0.2$ to 9$)$, with $43(68 \%)$ of high-grade, five intermediate grade $(8 \%)$ and 15 low grade $(24 \%)$. The tumour was located in a digit in 16 patients $(25 \%)$, the palm in $27(43 \%)$, the carpal area (including the carpal tunnel) in $14(22 \%)$ and the dorsum in six $(10 \%)$. We were only able to assess the depth of the tumours in one centre where the data was routinely recorded in the database. In this group, 15 of the 33 (45\%) tumours were classified as being deep and $18(55 \%)$ as subcutaneous.

Of the 63 patients, $24(38 \%)$ had their initial surgery elsewhere. An incisional biopsy had been carried out in 14 patients and an unplanned attempt at excision in ten. These patients were restaged after referral and then underwent their definitive surgical treatment.

All 63 patients underwent surgery. Six patients (10\%) had a radical below-elbow amputation, 12 (19\%) underwent partial amputation (nine ray and three digit) and 45 (71\%) underwent attempted wide local excision. The margins of excision were clear in 44 patients $(70 \%)$, involved in seven $(11 \%)$ and were unreported in $12(19 \%)$, all of whom had undergone local excision. All 18 patients who had undergone amputation had clear margins, but 19 of the $45(42 \%)$ with a local excision had involved margins. There was no association between the status of the surgical margin and the grade of the tumour.

Post-operative radiotherapy was used for 23 patients $(36.5 \%)$ and was given to 8 of the 14 patients $(57 \%)$ with high or intermediate grade tumours and involved margins, 
Table II. Univariate analysis for prognostic factors on the development of local recurrence

\begin{tabular}{|c|c|c|}
\hline Variables & Five-year risk of local recurrence (\%) & p-value \\
\hline \multicolumn{3}{|l|}{ Gender } \\
\hline Male & 19 & \\
\hline Female & 44 & 0.46 \\
\hline \multicolumn{3}{|l|}{ Age (yrs) } \\
\hline$<50$ & 17 & \\
\hline$\geq 50$ & 42 & 0.57 \\
\hline \multicolumn{3}{|l|}{ Tumour size $(\mathrm{cm})$} \\
\hline$\leq 4$ & 17 & \\
\hline$>4$ & 29 & 0.27 \\
\hline \multicolumn{3}{|l|}{ Histological grade } \\
\hline Low and intermediate & 19 & \\
\hline High & 48 & 0.49 \\
\hline \multicolumn{3}{|l|}{ Histological diagnosis } \\
\hline Clear cell sarcoma & 60 & 0.03 \\
\hline Epithelioid sarcoma & 48 & 0.96 \\
\hline Synovial sarcoma & 43 & 0.72 \\
\hline Other diagnoses & 21 & \\
\hline Previous excision/advertent biopsy & 25 & \\
\hline Primary treatment & 29 & 0.28 \\
\hline \multicolumn{3}{|l|}{ Surgical margin } \\
\hline Wide/marginal (clear) & 14 & \\
\hline Intralesional (involved) & 80 & $<0.0001$ \\
\hline \multicolumn{3}{|l|}{ Type of surgery } \\
\hline Local excision & 50 & \\
\hline Amputation & 8 & 0.005 \\
\hline \multicolumn{3}{|l|}{ Adjuvant radiotherapy } \\
\hline Yes & 42 & \\
\hline No & 24 & 0.02 \\
\hline
\end{tabular}

and in 12 of the $34(35 \%)$ patients with high or intermediate grade tumours and clear margins. Radiotherapy was also used in three patients with low grade tumours, two of the five with involved margins and one of the eight with clear margins. Two patients had adjuvant chemotherapy for synovial sarcoma, while one patient with a high-grade alveolar rhabdomyosarcoma had pre-operative chemotherapy.

Local recurrence. There were 20 patients (32\%) who developed a local recurrence at a mean of 21 months (1 to 87). The five- and ten-year risk of local recurrence for the cohort of 63 patients was $37 \%$ and $52 \%$, respectively. Table II shows the univariate analysis of prognostic factors for local recurrence for all patients.

The type of surgery performed (amputation or local excision) had a highly significant impact on local control. Only one of the 18 patients who underwent amputation developed a local recurrence (6\%) compared with $42 \%$ (19 of 45 patients) of those undergoing attempted limb salvage $(\mathrm{p}=0.005)$. An involved margin was the most highly significant risk factor for local recurrence $(\mathrm{p}<0.0001)$ (Fig. 2).
Previous biopsy or inadvertent excision did not significantly increase the risk of local recurrence $(\mathrm{p}=0.28)$.

The effect of radiotherapy in improving local control was difficult to assess but local recurrence occurred in nine of the ten patients $(90 \%)$ who had involved margins and who received radiotherapy and in four of the nine (44\%) with involved margins but who did not receive radiotherapy. Of the 13 patients with clear margins who received radiotherapy, three $(23 \%)$ developed a local recurrence compared with 4 of $31(13 \%)$ of those with clear margins who did not receive radiotherapy.

In the 45 patients undergoing limb salvage, we identified three tumour types which had a greater than $50 \%$ risk of local recurrence (epithelioid sarcoma 60\%, synovial sarcoma $57 \%$ and clear cell sarcoma 55\%). This is compared with a $27 \%$ risk for all other types of tumour. The significance of marginal status was even more apparent in this group. The risk of local recurrence was $74 \%$ in those with an involved margin compared with $26 \%$ in those with a clear margin. The addition of radiotherapy made no appreciable difference as two of the six patients (33\%) with an 


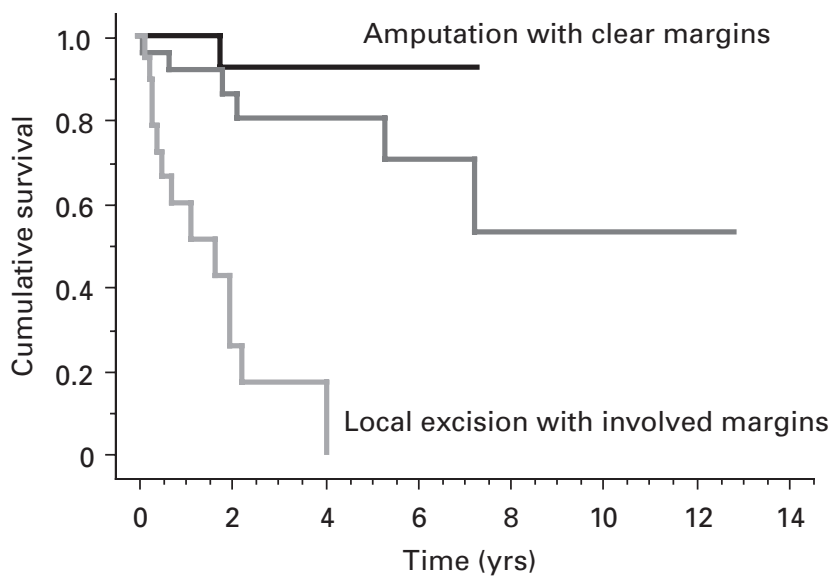

Fig. 2

Kaplan-Meier survival curve showing the local control rate of patients with respect to surgical treatment and margins of excision $(p<0.0001)$.

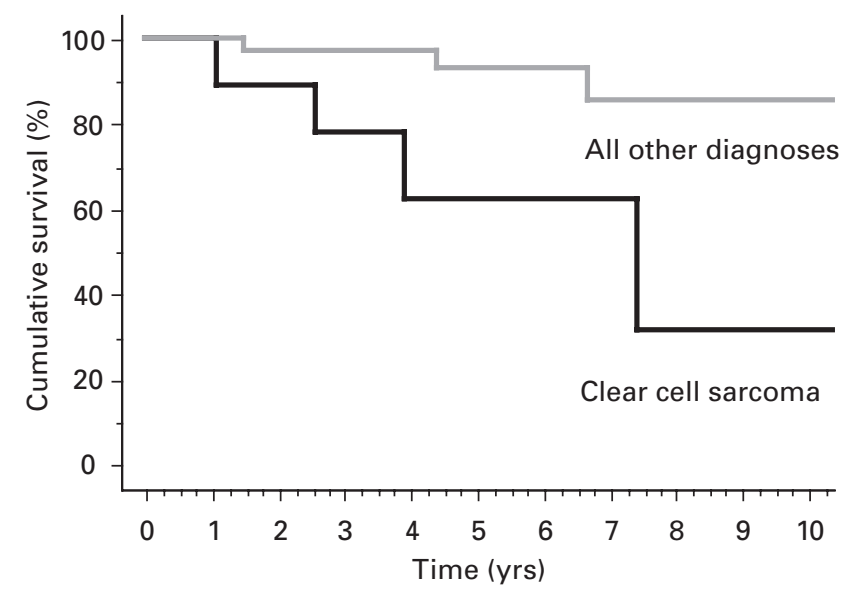

Fig. 4

Kaplan-Meier survival curve showing the overall five-year survival of patients with respect to a diagnosis of clear cell sarcoma $(p=0.006)$.

involved margin who received radiotherapy developed a local recurrence.

The only factor that remained significant on multivariate analysis was an involved margin. Patients with an involved margin had a 12 times greater risk of local recurrence than those with a clear margin (95\% confidence interval (CI) 4.1 to $35.1 ; \mathrm{p}<0.0001)$.

Distant failure. Metastases developed in 14 patients $(22 \%)$ after a mean of 41 months (4 to 147). They occurred in 13 after local excision and one after amputation $(\mathrm{p}=0.042)$. The risk of distant failure in this series was $26 \%$ at five years and $47 \%$ at ten years. The most significant risk fac-

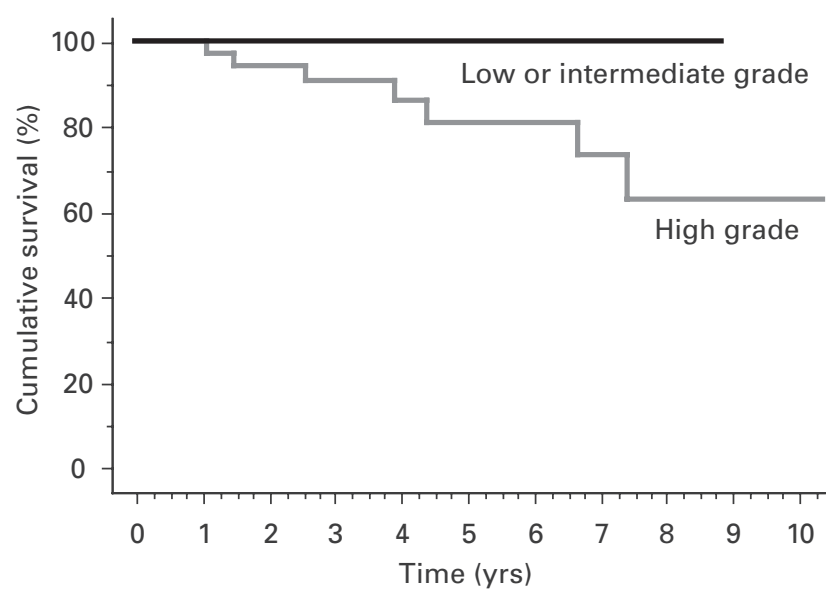

Fig. 3

Kaplan-Meier survival curve showing the overall five-year survival of patients with respect to grade of the tumour $(p=0.03)$.

tors on univariate and multivariate analysis were grade of tumour $(\mathrm{p}=0.02)$ and marginal status $(\mathrm{p}=0.002)$. Highgrade tumours were four times more likely to metastasise than low or intermediate grade tumours. Patients with an involved margin had a $42 \%$ risk of developing metastases compared with those with clear margins who had a $13 \%$ risk. Patients with epithelioid sarcoma and clear cell sarcoma were at risk of developing lymph node metastases higher up the arm whilst all other metastases were in the lungs. Six of the 11 patients with clear cell sarcoma had developed metastases by the time of this study.

Of the 43 patients with high-grade tumours, 14 (32.5\%) were treated by amputation, of whom one developed a local recurrence and one metastases; 12 (86\%) remained disease free. Of the 29 treated by local excision, 14 (48\%) developed local recurrence. Nine of these subsequently developed metastases and a further three developed metastases without local recurrence. Only 12 of these 29 patients $(41 \%)$ remained disease free $(\mathrm{p}=0.006)$.

Overall survival. At follow-up eight patients $(12.7 \%)$ had died of disease, two $(3 \%)$ had died of other causes, six $(9.5 \%)$ are alive with metastases and $47(74.8 \%)$ are alive with no evidence of disease. The overall survival for the entire cohort of 63 patients was $87 \%$ at five years and $71 \%$ at ten years. Overall survival was related to grade $(\mathrm{p}=0.03)$ (Fig. 3), size $(\mathrm{p}=0.001)$ of tumour and marginal status $(\mathrm{p}=0.02)$. All the tumour-related deaths were in patients with high-grade tumours and none of the patients with low or intermediate grade tumours have died to date. Four of the deaths arose in patients with clear cell sarcoma, which carried the poorest survival rate (Fig. 4) and a five-year survival of $62 \%$ compared with $92 \%$ in those with other histological subtypes $(\mathrm{p}=0.006)$. Only one $(5.5 \%)$ of the 18 patients who had an amputation subsequently died, com- 
Table III. Univariate analysis for prognostic factors of overall survival

\begin{tabular}{|c|c|c|}
\hline Variables & Five-year survival rate (\%) & p-value \\
\hline \multicolumn{3}{|l|}{ Gender } \\
\hline Male & 89 & \\
\hline Female & 81 & 0.91 \\
\hline \multicolumn{3}{|l|}{ Age (yrs) } \\
\hline$<50$ & 97 & \\
\hline$\geq 50$ & 70 & 0.18 \\
\hline \multicolumn{3}{|l|}{ Tumour size $(\mathrm{cm})$} \\
\hline$\leq 4$ & 95 & \\
\hline$>4$ & 61 & 0.001 \\
\hline \multicolumn{3}{|l|}{ Histological grade } \\
\hline Low and intermediate & 100 & \\
\hline High & 80 & 0.03 \\
\hline \multicolumn{3}{|l|}{ Histological diagnosis } \\
\hline Clear cell sarcoma & 62 & 0.006 \\
\hline Epithelioid sarcoma & 100 & 0.16 \\
\hline Synovial sarcoma & 84 & 0.86 \\
\hline Previous excision/advertent biopsy & 89 & \\
\hline Primary treatment & 84 & 0.59 \\
\hline \multicolumn{3}{|l|}{ Surgical margin } \\
\hline Clear & 95 & \\
\hline Involved & 60 & 0.02 \\
\hline \multicolumn{3}{|l|}{ Type of surgery } \\
\hline Local excision & 86 & \\
\hline Amputation & 86 & 0.55 \\
\hline \multicolumn{3}{|l|}{ Adjuvant radiotherapy } \\
\hline Yes & 82 & \\
\hline No & 90 & 0.42 \\
\hline
\end{tabular}

pared with seven $(15.5 \%)$ of the 45 who had a local excision $(\mathrm{p}=0.55)$. Neither adjuvant radiotherapy $(\mathrm{p}=0.42)$ nor treatment prior to referral affected survival $(\mathrm{p}=0.59)$. Although there was a trend for patients who developed local recurrence to have a poorer survival $(82 \%$ at five years for those who developed local recurrence $v s 90 \%$ for those without) this did not reach significance. The best prognostic indicator was a combination of size and grade of tumour with a Kaplan-Meier survivorship of $0 \%$ at eight years in the group with high-grade tumours larger than 4 $\mathrm{cm}$ at diagnosis, compared with $96 \%$ survival for all other tumours. Table III shows the factors associated with overall survival.

\section{Discussion}

Soft-tissue sarcomas of the hand comprise a very small proportion of tumours in the extremities. ${ }^{18}$ Their incidence, assuming that they comprise $2 \%$ of all soft-tissue sarcomas, would suggest perhaps one per 1250000 population per year. They are usually small, painless lesions at presentation, often with no particular characteristic features. They tend to be aggressive and carry a worse prognosis than sim- ilarly sized tumours in other anatomical regions. ${ }^{19,20}$ The histological spectrum of soft-tissue sarcomas in the hand is limited and uncommon subtypes tend to predominate. ${ }^{20}$ In our series, the most frequent subtypes were synovial sarcoma, clear cell sarcoma and epithelioid sarcoma. Owens et $\mathrm{al},{ }^{13}$ have reported synovial sarcoma to be the most common lesion in the hand. This is supported by the Memorial Sloan-Kettering Cancer Centre series. ${ }^{12}$

A significant proportion of these tumours undergo unplanned surgery before referral to a specialist oncological centre. In this study, $38 \%$ of the patients had either an unplanned excision or a biopsy. This figure is lower than that seen in previous studies. Bray et $\mathrm{al}^{2}$ found $68 \%$ of patients with distal upper extremity soft-tisue sarcomas had received previous treatment while Lin et al, ${ }^{7}$ found $95 \%$ of patients with soft-tissue sarcomas in the hand and foot were referred after previous surgery. An unplanned excision can lead to difficulty in obtaining wide margins at re-excision which increases the risk of local recurrence. ${ }^{8,9,21}$ However, we found no significant correlation between previous surgical excision and risk of local recurrence or overall survival. 
The biggest problem with soft-tissue sarcomas of the hand is their tendency to spread rapidly between compartments which makes excision with a wide margin difficult. As a result, the surgeon and the patient are often faced with the dilemma of preserving function at the expense of clear margins or of obtaining clear margins by carrying out an amputation.

We have shown that compromising surgical margins results in a 12 times greater risk of local recurrence, a three times greater risk of developing metastases and a five times greater risk of death than for those with clear margins.

Positive margins have been shown to increase the risk of local recurrence in extremity soft-tissue sarcomas in several studies. ${ }^{21-23}$ Lin et $\mathrm{al}^{7}$ and Brien et al, ${ }^{12}$ demonstrated this specifically for soft-tissue sarcomas of the hand. In our study 19 of the $20(95 \%)$ recurrences were seen in patients who had a limited excision rather than an amputation. Local recurrence did not correlate significantly with size or grade of tumour.

We were unable to identify any convincing benefit of post-operative radiotherapy, but this is undoubtedly because of the small number of patients and selection bias. Radiotherapy was only given to patients who were thought to carry the highest risk of local recurrence. These results are similar to those of Brien et $\mathrm{al}^{12}$ who reviewed 23 patients and showed that the risk of local recurrence in the presence of positive margins could not be negated by radiotherapy. Lin et al, ${ }^{7}$ showed that radiation improved local control in patients who did not undergo re-excision but not in patients who underwent a re-excision with negative margins. Jyothirmayi et al, ${ }^{10}$ showed that patients treated with radiotherapy after surgery for local recurrence had a higher rate of local failure compared with those treated at initial presentation.

We have shown that local control is clearly related to the histological diagnosis, particularly when attempts are made at limb salvage. This is reflected by the high risk of local recurrence in the three tumour types, clear cell sarcoma, epithelioid sarcoma and synovial sarcoma. Chung and Enzinger ${ }^{24}$ suggest that clear cell sarcoma carries a poor prognosis with most tumours recurring within four years of treatment and nearly $50 \%$ of patients dying of metastases. Prat, Woodruff and Marcove, ${ }^{25}$ reported a local recurrence rate of $63 \%$ and a $58 \%$ rate of metastases in patients with epithelioid sarcoma.

In our series, patient survival was $87 \%$ at five years and $71 \%$ at ten years, which seems comparable to previous studies. Lin et $\mathrm{al}^{7}$ reported a disease-specific survival of $76 \%$ and $65 \%$ at five and ten years for 84 patients with localised hand and foot soft-tissue sarcomas. The survival of patients in our study was directly related to tumour size and grade, it is essential to obtain a clear surgical margin. Similar results have been shown in previous studies.,14
We have not addressed the functional differences between treatments in this study, but it is certainly a subject worthy of attention, particularly the question of whether the increased risks of limb salvage in patients with involved margins is ever justified.

No benefits in any form have been received or will be received from a commercial party related directly or indirectly to the subject of this article.

\section{References}

1. Ferguson PC. Surgical considerations for management of distal extremity soft tissue sarcomas. Curr Opin Oncol 2005:17:366-9.

2. Bray PW, Bell RS, Bowen CV, Davis A, O'Sullivan B. Limb salvage surgery and adjuvant radiotherapy for soft tissue sarcomas of the forearm and hand. J Hand Surg [Am] 1997;3:495-503.

3. Keus RB, Rutgers EJ, Ho GH, et al. Limb-sparing therapy of extremity soft tissue sarcomas: treatment outcome and long-term functional results. Eur J Cancer 1994;30:1459-63.

4. Pignatti G, Campanacci M. Surgical approach to soft tissue sarcomas of the extremities. Ann Oncol 1992;3 (Suppl 2):59-61.

5. Rosenberg SA, Tepper J, Glatstein E, et al. The treatment of soft-tissue sarcomas of the extremities: prospective randomized evaluations of (1) limbsparing surgery plus radiation therapy compared with amputation and (2) the role of adjuvant chemotherapy. Ann Surg 1982;196:305-15.

6. Freeza G, Barbieri E, Ammendolia I, et al. Surgery and radiation therapy in the treatment of soft tissue sarcomas of extremities. Ann Oncol 1992;3(Suppl 2):93-5.

7. Lin PP, Guzel VB, Pisters PW, et al. Surgical management of soft tissue sarcomas of the hand and foot. Cancer 2002;95:852-61.

8. Kawai A, Hasizume H, Sugihara S, Morimoto Y, Inoue H. Treatment of bone and soft tissue sarcomas of the hand and wrist. Int Orthop 2002;26:26-30.

9. Gustafson P, Arner M. Soft tissue sarcoma of the upper extremity: descriptive data and outcome in a population-based series of 108 adult patients. J Hand Surg [Am] 1999;24:668-74

10. Jyothirmayi R, Sittampalam Y, Harmer C. Soft tissue sarcoma of the hand or foot: conservative surgery and radiotherapy. Sarcoma 1999;3:17-24

11. Karakousis CP, De Young C, Driscoll DL. Soft tissue sarcomas of the hand and foot: management and survival. Ann Surg Oncol 1998;5:238-40.

12. Brien EW, Terek RM, Geer RJ, et al. Treatment of soft-tissue sarcomas of the hand. J Bone Joint Surg [Am]1995;77-A:564-71.

13. Owens JC, Shiu MH, Smith R, Hajdu SI. Soft tissue sarcomas of the hand and foot. Cancer 1985;55:2010-18

14. McPhee M, McGrath BE, Zhang $\mathbf{P}$, et al. Soft tissue sarcoma of the hand. J Hand Surg $[A m]$ 1999;5:1001-7.

15. Trojani M, Contesso G, Coindre JM, et al. Soft-tissue sarcomas of adults; study of pathological prognostic variables and definition of a histopathological grading system. Int J Cancer 1984;33:37-42.

16. Kaplan EL, Meier P. Nonparametric observations from incomplete observations. J Am Stat Assoc 1958;53:457-81.

17. Cox DR. Regression models and life tables. J Roy Stat Soc 1972;34:187-220.

18. Buecker PJ, Villafuerte JE, Hornicek FJ, Gebhardt MC, Mankin HJ. Improved survival for sarcomas of the wrist and hand. J Hand Surg [Am]2006;31:452-5.

19. Campanacci M, Bertoni F, Laus M. Soft tissue sarcoma of the hand. Ital J Orthop Traumatol 1981;7:313-27.

20. Rosenberg AE, Schiller AL. Soft tissue sarcomas of the hand. Hand Clin 1987;3:247-61.

21. Gerrand CH, Wunder JS, Kandel RA, et al. Classification of positive margins after resection of soft-tissue sarcoma of the limb predicts the risk of local recurrence. $J$ Bone Joint Surg [Br] 2001;83-B:1149-55.

22. Stojadinovic A, Leung DH, Hoos A, et al. Analysis of the prognostic significance of microscopic margins in 2084 localized primary adult soft tissue sarcomas. Ann Surg 2002;235:424-34

23. Vraa S, Keller J, Nielson OS, et al. Prognostic factors in soft tissue sarcomas: the Aarhus experience. Eur J Cancer 1998;34:1876-82.

24. Chung EB, Enzinger FM. Malignant melanoma of soft parts: a reassessment of clear cell sarcoma. Am J Surg Pathol 1983;7:405-13.

25. Prat J, Woodruff JM, Marcove RC. Epithelioid sarcoma: an analysis of 22 cases indicating the prognostic significance of vascular invasion and regional lymph noted metastasis. Cancer 1978;41:1472-87. 